\title{
Autoeficacia académica y rendimiento escolar: un estudio metodológico y correlacional en escolares
}

Patricio Galleguillos Herrera. Universidad de Granada

Eva Olmedo Moreno. Universidad de Granada

Recepción: 23 de marzo de 2017 | Aceptado: 24 de marzo de 2017 Correspondencia: Eva Olmedo | Correo-e: emolmedo@ugr.es

\section{iD 0000-0003-0558-1513}

Citar: Galleguillos Herrera, P. y Olmedo Moreno, E. (2017). Autoeficacia académica y rendimiento escolar: un estudio metodológico y correlacional en escolares. ReiDoCrea, 6, 156169.

Resumen: El presente estudio tuvo como principal propósito establecer la relacional entre las creencias de autoeficacia percibida por los estudiantes de enseñanza básica y el rendimiento escolar obtenido en el periodo académico anterior (2015). Para tal efecto, se contó con la participación de 802 estudiantes (hombres y mujeres), de enseñanza básica y media. A nivel metodológico se aplicó la Escala de Autoeficacia Académica de los escolares (ACAES) la cual mostró, en la muestra de este estudio, adecuados índices psicométricos tanto a nivel factorial $(49,457 \%$ de varianza explicada) como de consistencia interna (alfa de Cronbach $=0,89$ ). Los resultados evidencian que existe una correlación positiva y significativa $(, 398)$ entre la autoeficacia y el rendimiento escolar, es decir, aquellos estudiantes con altos niveles de autoeficacia muestran altos promedios de rendimiento escolar. Por tanto, se consolida la idea que los procesos cognitivos y metacognitivos son fundamentales para los aprendizajes, por cuanto permiten el desarrollo de prácticas autoregulatorias que garantizan el logro de objetivos académicos.

Palabras clave: Autoeficacia | Rendimiento Escolar | Análisis Factorial Exploratorio

Academic Self-efficacy and School Performance: A Methodological and Correlational Study in Schools

Abstract: The main purpose of this paper is to define the relationship between the Self-efficacy beliefs perceived by the students of primary education and their academic performance during the previous academic period (2015). For this research study, 802 primary and secondary students (men and women) participated. In terms of methodology, the academic self-efficacy scale for students (ACAES) was administered which shows, in the sample of this study, sufficient psychometric rates, both at the factorial level $(49.457 \%$ of variance explained) and in the internal consistency (Cronbach's Alfa $=0.89$ ). The results demonstrate a significant positive correlation (.398) between self-efficacy and academic performance; in other words, those students with high levels of self-efficacy show high averages of academic performance. Therefore, the idea that cognitive and metacognitive procedures are fundamental for learning is consolidated, because they allowing the development of self-regulation practices that guarantee the achievement of academics goals.

Key Words: Self-efficacy | Academic Performance | Exploratory Factor Analysis

\section{Introducción}

Constantemente, el ser humano busca mejorar el desempeño de sus acciones para el logro de objetivos personales, académicos, laborales, sociales, entre otros.

En las teorías sobre motivación humana, según Camposeco (2011), abundan propuestas que se centran en este aspecto. En muchos casos, el nivel de motivación, los estados afectivos y las conductas personales se basan más en lo que el sujeto piensa sobre las situaciones, que en la realidad objetiva.

En este sentido, Velásquez (2012) plantea que a fines de la década de los setenta, se propone la teoría de la autoeficacia, siendo ésta producto de dos ideas que Albert 
Bandura tiene en mente, la primera hace referencia a que las intervenciones que modifican el ambiente son eficaces para modificar la conducta y la segunda que la intervención cognitiva del sujeto es imprescindible. Se avanza entonces a identificar a la autoeficacia como un estado psicológico en el cual la persona evalúa su capacidad y habilidad de ejecutar determinada tarea, actividad, conducta, entre otros, en una situación específica con un nivel de dificultad previsto (Bardales, Díaz, Jiménez, Terreros y Valencia, 2006).

Bandura (1977, 1982, 1987, 1993, 1997, 1999 y 2001) fundamenta sus postulados de la autoeficacia en la teoría del aprendizaje social, la cual surge como respuesta a la visión conductista de la época y su poca efectividad para explicar los procesos por los que se adquiere y modifica la conducta social.

Desde los postulados teóricos de este autor, la autoeficacia se define como aquellos pensamientos de una persona referidos a su capacidad para organizar y ejecutar los cursos de acción necesarios para conseguir determinados logros. Según este autor, los eventos sobre los que se ejerce esta influencia son muy variados, pudiendo aplicarse a procesos de pensamiento, a estados afectivos, a la puesta en marcha de acciones, al cambio de las condiciones ambientales o a la autorregulación de la motivación.

Por tanto, para Bandura (1987) la autoeficacia incluye "los juicios de cada individuo sobre sus capacidades, en base a los cuales organizará y ejecutará sus actos de modo que le permitan alcanzar el rendimiento deseado". En el ámbito académico, Bandura (1995) se refiere a las creencias de autoeficacia, como las creencias de los estudiantes respecto a su eficacia para regular su propio aprendizaje y dominar los diversos temas académicos, que operan sobre el rendimiento de dichos estudiantes.

Desde esta conceptualización se puede apreciar la importancia de la autoeficacia sobre la motivación y el aprendizaje, las percepciones de los individuos sobre sí mismos y sobre sus capacidades, constituyendo factores importantes en sus desempeños académicos.

De esta manera, según Contreras, Espinosa, Esguerra Pérez, Haikal, Polanía y Rodriguez (2005) la investigación ha demostrado que la autoeficacia resulta predictiva del logro académico; afectando la conducta del individuo en las elecciones que realiza, el esfuerzo que aplica, la perseverancia ante los obstáculos con que se enfrenta, los patrones de pensamiento y las reacciones emocionales que experimenta.

Siguiendo a nivel académico, Cartagena (2008) menciona que para aprender es necesario: (a) que el alumno sea cognitivamente capaz de enfrentarse las tareas de aprendizaje y (b) que se encuentre motivacionalmente orientado hacia el aprendizaje o, al menos, hacia la resolución efectiva de dichas tareas.

En este sentido surgen las expectativas personales, como un proceso importante en los estudios a nivel psicológico y su relación con diferentes áreas de desarrollo, entre ellas el rendimiento académico. Para Pajares y Schunk, (2001) la autoeficacia tiene un papel vital en el ámbito académico por cuanto se ha evidenciado que un buen desempeño académico no puede ser garantizado solo por los conocimientos y habilidad de los individuos. Las creencias de eficacia pueden determinar un desempeño diferente en dos personas con el mismo grado de habilidad. Esto se debe a que el éxito académico demanda procesos reguladores como la autoevaluación, el automonitoreo y el uso de estrategias metacognitivas de aprendizaje, procesos que 
son influidos positivamente por un alto grado de creencia en la propia capacidad o autoeficacia.

Bandura (1986) plantea que la autoeficacia en un campo concreto implica una capacidad generativa en la que es necesario integrar subcompetencias cognitivas, sociales y conductuales en actuaciones encaminadas a alcanzar determinados propósitos. Por el contrario, para Bandura (1987), en general, las personas que dudan de sus capacidades:

(...) evitan las tareas difíciles, reducen sus esfuerzos y se dan rápidamente por vencidos frente a las dificultades, acentúan sus deficiencias personales, disminuyen sus aspiraciones y padecen en gran medida ansiedad y estrés. Tales dudas sobre sí mismo disminuyen el rendimiento y generan gran malestar (...) las investigaciones realizadas demuestran que el individuo que se considera a sí mismo altamente eficaz, actúa, piensa y siente de forma distinta de aquel que se percibe ineficaz (p. 420).

Según González Fernández (2005), las creencias de autoeficacia actúan en el momento en que el alumno ingresa al salón de clases, ya sea, en sus expectativas de éxito ante los objetivos propuestos, en el control de los resultados obtenidos o en las causas mediante las cuales explican esos resultados.

Por tal motivo, Paoloni y Bonetto (2013) resumen que las creencias sobre uno mismo, particularmente las que refieren a percepciones de competencia, constituyen un aspecto relevante en las investigaciones sobre motivación académica y en el desempeño vinculado con los éxitos y fracasos de los estudiantes en contextos de aprendizaje (Contreras, Espinosa, Esguerra Pérez, Haikal, Polanía y Rodriguez, 2005; Paoloni, Rinaudo, Donolo y Chiecher, 2006).

En este sentido, Serra (2010) plantea que "en su investigación Contreras et al., (2005) y Serra (2010) observaron que el aprovechamiento académico, está directa y positivamente influenciada por el nivel de percepción de autoeficacia en estudiantes. Esto se debe a que los estudiantes con un alto sentido de eficacia para completar una tarea educativa participarán con mayor disposición, se esforzarán y persistirán durante más tiempo que aquellos que dudan de sus capacidades ante las dificultades (Bandura, 1977; Multon et al., 1991). Además, se señala que el tamaño del efecto de la persistencia puede ser reflejo del hecho de que un alto sentido de la eficacia puede fomentar la persistencia en tareas difíciles, pero puede reducirla mediante las soluciones rápidas cuando las tareas son sólo moderadamente difíciles. Multon et al. (1991) encontró una relación más fuerte entre las creencias de eficacia y el logro de los estudiantes cuyo progreso académico era bajo $(r=0.56)$ que entre jóvenes con un buen progreso académico $(r=0.33)$ ".

Son múltiples los estudios sobre autoeficacia y rendimiento académico. Al respecto, Carrasco y Del Barrio (2002) señalan que la autoeficacia está siendo estudiada en el ámbito infantil y juvenil de forma intensa y en relación con el funcionamiento académico (Bandura, 1993; Bandura, Barbaranelli, Caprara y Pastorelli, 1996; Bong, 2001), el desarrollo intelectual (Schunk, 1989; Zimmerman, Bandura y Martínez-Pons, 1992) y la creatividad entre otros (Zimmerman y Bandura, 1994).

En todos estos estudios los niveles de autoeficacia percibida han constituido una variable fundamental que afecta a la socialización y al éxito del niño. Un elevado nivel de autoeficacia percibida se ha mostrado como un elemento protector que hace aumentar la motivación y la consecución académica (Bandura et al., 1996), disminuir las alteraciones emocionales (Villamarín, 1990a), al tiempo que mejora las conductas 
saludables en el cuidado físico (Villamarín, 1990b), la prevención de conductas de riesgo sexual (Gómez, Ibaceta, Muñoz y Pardo, 1996) y la mayor tolerancia al dolor (Rejeski, Craven, Ettinger, McFarlane y Shumaker, 1996). Otro ámbito de mejoría se percibe en el descenso de conductas antisociales (Caprara, Scabini, Barbaralli, Pastorelli, Regalia y Bandura, 1998; Bandura, Caprara, Barbaranelli, Pastorelli y Regalia, 2001).

En suma, Bandura (1997) y Zimmerman (1995) plantean que para apoyar en la formación a los estudiantes se debe no solo atender la enseñanza de destrezas de trabajo intelectual, sino desarrollar en los estudiantes creencias y capacidades de autorregulación que les permita controlar sus aprendizajes a lo largo de toda la vida.

Para tal efecto, es necesario desarrollar un ciclo de aprendizaje autorregulatorio que permita a los estudiantes observar y autoevaluar su efectividad en el estudio, establecer metas y utilizar estrategias de aprendizaje, así como supervisar y refinar esas estrategias en función de los resultados académicos obtenidos. Este ciclo de aprendizaje autorregulatorio otorga a los estudiantes un sentido de control personal del aprendizaje, que es una de las fuentes principales de motivación intrínseca para continuar aprendiendo durante toda la vida.

En síntesis, para Poloni y Bonetto (2013) los antecedentes revisados reflejan que las creencias de autoeficacia están relacionadas de manera recíproca con el desempeño y el logro académico obtenido (Paoloni et al., 2006). Concretamente, se considera que cuanto mayor es la eficacia percibida, más desafiantes serán las metas que las personas establezcan para sí, más firme el esfuerzo, la persistencia y el compromiso para con las mismas, y mayores las posibilidades de activar emociones placenteras y obtener resultados de calidad en la tarea asumida. Por otra parte, los progresos observados hacia la meta o los logros obtenidos, fortalecen en los sujetos su percepción de autoeficacia (González Fernández, 2005; Huertas, 1997; Paoloni et al., 2006) e incluso, un bajo nivel de autoeficacia puede ser responsable, entre otras causas, de la disminución del interés del alumno hacia el estudio (Hackett, 1995).

\section{Método}

\section{Participantes}

El presente estudio trabajó con un total de 802 estudiantes. Del total un $61.3 \%$ pertenecen al ciclo básico de educación ( $5^{\circ}$ a $8^{\circ}$ de enseñanza básica) y un $38.7 \%$ pertenece al ciclo media de educación (I a IV de enseñanza media). De igual forma, un $42.4 \%$ corresponde a establecimientos Particular Subvencionados y un $57.6 \%$ a establecimientos Particular Privado, todos de la Comuna de Viña del Mar - Chile.

\section{Instrumento}

El instrumento para la recogida de datos corresponde a la Escala de Autoeficacia de los Escolares (ACAES), la cual define la variable autoeficacia como "las creencias (conductas, cogniciones y afectos) de los estudiantes acerca de sus propias capacidades para el logro de determinados resultados académicos (actividades/dominios)". La escala consta de 18 afirmaciones a ser valoradas en un continuo de 1 a 5 desde "Nunca puedo" a "Siempre puedo". Las afirmaciones se agrupan empíricamente en tres factores, a saber, CONFIANZA EN EL DESEMPEÑO DE LA TAREA; ESFUERZO EN LA REALIZACIÓN DE LA TAREA y COMPRENSIÓN DE LA TAREA. Cabe destacar, que estos tres factores surgen del análisis factorial exploratorio realizado a los datos y cuyo resultado corresponde a la estructura teoría 
con mayor parsimonia que permita interpretar los hallazgos y establecer su relación con la variable rendimiento escolar, la cual se recoge a través del promedio declarado por cada participante, para el año 2015.

\section{Procedimiento}

Se contactó, a través de la Secretaría Ministerial de Educación de la Región de Valparaíso, a los directores de los centros educativos para solicitar acceso a la muestra, Dichas autoridades tomaron conocimiento del estudio, en base a reunión presencial con un autor del estudio, para luego autorizar por escrito la aceptación de participar. Cada establecimiento educacional designó a un encargado quien fue la contraparte del equipo investigador para recibir la capacitación necesaria en la aplicación del instrumento. El instrumento fue aplicado durante el segundo semestre académico 2016. La aplicación se realizó colectivamente, dentro de cada establecimiento y en horario de clases.

\section{Análisis de datos}

El procesamiento de datos se realizó por medio del programa denominado SPSS (versión 15.0), paquete estadístico considerado idóneo para trabajos en Ciencias Sociales. Los datos fueron tabulados y posteriormente se realizó el análisis factorial exploratorio para componentes principales y rotación varimax. Para el nivel de consistencia interna se realizó el análisis Alfa de Cronbach). Las correlaciones entre cada factor explorado y el rendimiento escolar declarado se realizaron con la prueba de Spearman Brown. Los ciclos educacionales fueron comparados a través de la prueba T de Student para muestras independientes.

\section{Resultados}

\section{Análisis de ítems}

La tabla $\mathrm{n}^{0} 1$ muestra el comportamiento estadístico de cada afirmación y el grado de asimetría de las mismas. Se observa que existe una predominancia de distribución asimétrica negativa donde los valores mayoritariamente se encuentran sobre el valor de la media. Siendo mayores su diferencia para los ítems $N^{0} 1$ "Trabajar con cualquier compañero y lograr buenas notas"; N² "Trabajar en cualquier tarea y lograr buenas notas"; No3 "Entender lo que enseña cualquier profesor" y N 14 "Coopera muy bien en los trabajos que realizo en grupo". Por otra parte, los ítems $N^{\circ} 5$ "Aportar buenas ideas para hacer mis tareas en todos los ramos"; N¹2 "repetir una tarea hasta lograr hacerlo bien"; №6 "Esforzarme mucho más que mis compañeros para que me vaya bien en todos los ramos"; $\mathrm{N}^{\circ} 8$ "Organizar mi tiempo para cumplir con todo lo que los profesores piden"; $N^{\circ} 9$ "Sacarme buenas notas en las pruebas que creo difíciles"; N¹0 "Estudiar más horas cuando tengo pruebas difíciles"; N¹2 "Repetir una tarea hasta lograr hacerlo bien"; №16 "Expresar mi opinión aunque no esté de acuerdo con lo que dice el profesor"; $\mathrm{N}^{\circ} 17$ "Entender lo que explica un profesor aunque exista desorden en la sala" y $\mathrm{N}^{0} 18$ "Estudiar primero, y luego hacer otras cosas", presentan una distribución normal de sus puntajes. 


\begin{tabular}{|c|c|c|c|c|c|}
\hline \multirow{2}{*}{\multicolumn{6}{|c|}{$\begin{array}{r}\text { Tabla N01: Estadística descriptiva } \\
\text { Estadisticos descriptivos }\end{array}$}} \\
\hline & & & & & \\
\hline & \multirow{2}{*}{$\begin{array}{c}\mathrm{N} \\
\text { Estadistico }\end{array}$} & \multirow{2}{*}{$\begin{array}{c}\text { Media } \\
\text { Estadístico } \\
\end{array}$} & \multirow{2}{*}{$\begin{array}{c}\text { Desv. típ. } \\
\text { Estadistico }\end{array}$} & \multicolumn{2}{|c|}{ Asimetria } \\
\hline & & & & Estadístico & Error típico \\
\hline $\begin{array}{l}\text { 1.Trabajar con cualquier } \\
\text { compañero y lograr } \\
\text { buenas notas }\end{array}$ & 801 & 3,92 & ,888 & $-1,074$ & ,086 \\
\hline $\begin{array}{l}\text { 2. Trabajar en cualquier } \\
\text { tarea y lograr buenas } \\
\text { notas }\end{array}$ & 799 & 3,87 & ,886 &,- 932 & ,086 \\
\hline $\begin{array}{l}\text { 4. Realizar bien cualquier } \\
\text { tarea que me den }\end{array}$ & 789 & 3,88 & ,909 &,- 841 & ,087 \\
\hline $\begin{array}{l}\text { 5.Aportar buenas ideas } \\
\text { para hacer mis tareas en } \\
\text { todos los ramos }\end{array}$ & 787 & 3,62 & 1,105 &,- 496 & ,087 \\
\hline $\begin{array}{l}\text { 6. Esforzarme mucho más } \\
\text { que mis compañeros } \\
\text { para que me vaya bien } \\
\text { en todos los ramos }\end{array}$ & 796 & 3,66 & 1,079 &,- 479 & ,087 \\
\hline $\begin{array}{l}\text { 14.Cooperar muy bien en } \\
\text { los trabajos que realizo } \\
\text { en grupo. }\end{array}$ & 789 & 4,22 & ,902 & $-1,268$ & ,087 \\
\hline $\begin{array}{l}\text { 15.Estudiar solo/a y rendir } \\
\text { muy bien en cualquier } \\
\text { actividad académica }\end{array}$ & 795 & 3,80 & 1,066 &,- 791 & ,087 \\
\hline $\begin{array}{l}\text { 16.Expresar mi opinión } \\
\text { aunque no esté de } \\
\text { acuerdo con lo que dice } \\
\text { el profesor }\end{array}$ & 797 & 3,60 & 1,267 &,- 579 & ,087 \\
\hline $\begin{array}{l}\text { 7. Realizar cualquier tipo } \\
\text { de tarea o trabajo que los } \\
\text { profesores den, aunque } \\
\text { sean dificiles }\end{array}$ & 799 & 3,81 & 1,050 &,- 742 & ,086 \\
\hline $\begin{array}{l}\text { 8. Organizar mi tiempo } \\
\text { para cumplir con todo lo } \\
\text { que los profesores piden }\end{array}$ & 799 & 3,42 & 1,222 &,- 422 & ,086 \\
\hline $\begin{array}{l}\text { 10.Estudiar más horas } \\
\text { cuando tengo pruebas } \\
\text { difíciles }\end{array}$ & 797 & 3,43 & 1,318 &,- 364 & 087 \\
\hline $\begin{array}{l}\text { 11.Esforzarme mucho } \\
\text { más para resolver tareas } \\
\text { difíciles }\end{array}$ & 785 & 3,75 & 1,127 &,- 725 & 087 \\
\hline $\begin{array}{l}\text { 12. Repetir una tarea } \\
\text { hasta lograr hacerlo bien }\end{array}$ & 785 & 3,50 & 1,243 &,- 513 & 087 \\
\hline $\begin{array}{l}\text { 18.Estudiar primero, y } \\
\text { luego hacer otras cosas } \\
\text { (jugar, ver tv) }\end{array}$ & 798 & 3,28 & 1,383 &,- 296 & , 087 \\
\hline $\begin{array}{l}\text { 3. Entender lo que enseña } \\
\text { cualquier profesor }\end{array}$ & 794 & 3,86 & ,907 &,- 983 & ,087 \\
\hline $\begin{array}{l}\text { 9. Sacarme buenas notas } \\
\text { en las prueba que creo } \\
\text { dificiles }\end{array}$ & 796 & 3,45 & 1,074 &,- 505 & , 087 \\
\hline $\begin{array}{l}\text { 13.Entender bien la idea } \\
\text { central que está } \\
\text { explicando el profesor o } \\
\text { lo que dice un libro }\end{array}$ & 797 & 3,87 & 1,003 &,- 837 & ,087 \\
\hline $\begin{array}{l}\text { 17.Entender lo que } \\
\text { explica un profesor } \\
\text { aunque exista desorden } \\
\text { en la sala. }\end{array}$ & 795 & 3,30 & 1,305 &,- 379 & ,087 \\
\hline N válido (según lista) & 703 & & & & \\
\hline
\end{tabular}

\section{Análisis factorial}

Previo al Análisis factorial se efectuó la medida de adecuación muestral KMO (KaiserMeyer- Olkin) la cual busca verificar si el tamaño de la muestra seleccionada se adecua y es suficiente para realizar los análisis, y la Prueba de Esfericidad de Bartlett la cual sólo permite realizar análisis factoriales con un valor máximo de 0,005. Los datos presentados en la Tabla $\mathrm{N}^{\circ} 2$ arrojaron un valor igual a 0,000 indicando que es aceptable realizar la aplicación del Análisis Factorial. 


\begin{tabular}{|c|c|c|}
\hline \multicolumn{3}{|c|}{ Tabla N²: Medidas de adecuación muestral } \\
\hline \multicolumn{3}{|c|}{ KMO y prueba de Bartlett } \\
\hline \multicolumn{2}{|c|}{$\begin{array}{l}\text { Medida de adecuación muestral de } \\
\text { Kaiser-Meyer-Olkin. }\end{array}$} & ,936 \\
\hline $\begin{array}{l}\text { Prueba de esfericidad } \\
\text { de Bartlett }\end{array}$ & $\begin{array}{l}\text { Chi-cuadrado } \\
\text { aproximado }\end{array}$ & 4040,959 \\
\hline & gl & 153 \\
\hline & Sig. &, 000 \\
\hline
\end{tabular}

La tabla $\mathrm{N}^{\circ} 3$ muestra el porcentaje de varianza explicada que se ha detectado con tres factores con autovalores iniciales iguales o superiores a 1. Se observa los ítems se agrupan en tres factores que en conjunto explican el $49.457 \%$ de la varianza.

\begin{tabular}{|c|c|c|c|c|c|c|c|c|c|}
\hline \multicolumn{10}{|c|}{ Tabla N³: Porcentaje de varianza explicada } \\
\hline \multicolumn{10}{|c|}{ Varianza total explicada } \\
\hline & \multicolumn{3}{|c|}{ Autovalores iniciales } & \multicolumn{3}{|c|}{$\begin{array}{c}\text { Sumas de las saturaciones al cuadrado } \\
\text { de la extracción }\end{array}$} & \multicolumn{3}{|c|}{$\begin{array}{c}\text { Suma de las saturaciones al cuadrado } \\
\text { de la rotación }\end{array}$} \\
\hline Componente & Total & $\begin{array}{c}\% \text { de la } \\
\text { varianza }\end{array}$ & $\%$ acumulado & Total & $\begin{array}{c}\% \text { de la } \\
\text { varianza }\end{array}$ & $\%$ acumulado & Total & $\begin{array}{c}\% \text { de la } \\
\text { varianza }\end{array}$ & $\%$ acumulado \\
\hline 1 & 6,564 & 36,468 & 36,468 & 6,564 & 36,468 & 36,468 & 3,482 & 19,343 & 19,343 \\
\hline 2 & 1,276 & 7,091 & 43,559 & 1,276 & 7,091 & 43,559 & 2,961 & 16,447 & 35,790 \\
\hline 3 & 1,062 & 5,898 & 49,457 & 1,062 & 5,898 & 49,457 & 2,460 & 13,667 & 49,457 \\
\hline 4 & ,924 & 5,136 & 54,593 & & & & & & \\
\hline 5 & ,830 & 4,610 & 59,202 & & & & & & \\
\hline 6 &, 772 & 4,289 & 63,491 & & & & & & \\
\hline 7 &, 733 & 4,072 & 67,563 & & & & & & \\
\hline 8 & ,706 & 3,924 & 71,487 & & & & & & \\
\hline 9 &, 642 & 3,568 & 75,055 & & & & & & \\
\hline 10 & ,616 & 3,424 & 78,479 & & & & & & \\
\hline 11 &, 590 & 3,280 & 81,759 & & & & & & \\
\hline 12 &, 560 & 3,110 & 84,869 & & & & & & \\
\hline 13 &, 521 & 2,896 & 87,764 & & & & & & \\
\hline 14 &, 489 & 2,717 & 90,481 & & & & & & \\
\hline 15 & ,469 & 2,605 & 93,086 & & & & & & \\
\hline 16 & ,453 & 2,515 & 95,601 & & & & & & \\
\hline 17 & ,439 & 2,439 & 98,041 & & & & & & \\
\hline 18 &, 353 & 1,959 & 100,000 & & & & & & \\
\hline
\end{tabular}

La tabla $\mathrm{N}^{0} 4$ muestra en forma agrupada la composición factorial final de los 18 ítemes. La agrupación efectuada se puede interpretar considerando los siguientes factores: FACTOR 1: Confianza en el desempeño de la tarea (afirmaciones $\mathrm{N}^{\circ}$ 1,2,4,5,6,14 y 15); FACTOR 2: Esfuerzo en la realización de la tarea (afirmaciones $\mathrm{N}^{0} 7,8,10,11,12,16$ y 18); FACTOR 3: Comprensión de la tarea (afirmaciones $\mathrm{N}^{\circ}$ 1, 2, 9 y 16). 
Tabla №4: Composición factorial del instrumento

Matriz de componentes rotados

\begin{tabular}{|c|c|c|c|}
\hline & \multicolumn{3}{|c|}{ Componente } \\
\hline & 1 & 2 & 3 \\
\hline $\begin{array}{l}\text { 1. Trabajar con cualquier } \\
\text { compañero y lograr } \\
\text { buenas notas }\end{array}$ & , 133 & ,674 & ,070 \\
\hline $\begin{array}{l}\text { 2. Trabajar en cualquier } \\
\text { tarea y lograr buenas } \\
\text { notas }\end{array}$ & ,354 & ,446 & ,434 \\
\hline $\begin{array}{l}\text { 4. Realizar bien cualquier } \\
\text { tarea que me den }\end{array}$ & ,367 & ,424 & ,340 \\
\hline $\begin{array}{l}\text { 5. Aportar buenas ideas } \\
\text { para hacer mis tareas en } \\
\text { todos los ramos }\end{array}$ & ,301 & ,633 & , 137 \\
\hline $\begin{array}{l}\text { 6.Esforzarme mucho más } \\
\text { que mis compañeros } \\
\text { para que me vaya bien } \\
\text { en todos los ramos }\end{array}$ & ,432 & ,459 & ,276 \\
\hline $\begin{array}{l}\text { 14.Cooperar muy bien en } \\
\text { los trabajos que realizo } \\
\text { en grupo. }\end{array}$ & ,226 & ,624 & ,006 \\
\hline $\begin{array}{l}\text { 15.Estudiar solo/a y rendir } \\
\text { muy bien en cualquier } \\
\text { actividad académica }\end{array}$ & ,322 & ,469 & ,357 \\
\hline $\begin{array}{l}\text { 16. Expresar mi opinión } \\
\text { aunque no esté de } \\
\text { acuerdo con lo que dice } \\
\text { el profesor }\end{array}$ &,- 057 &, 579 & ,238 \\
\hline $\begin{array}{l}\text { 7. Realizar cualquier tipo } \\
\text { de tarea o trabajo que los } \\
\text { profesores den, aunque } \\
\text { sean difíciles }\end{array}$ & ,460 & ,405 & ,305 \\
\hline $\begin{array}{l}\text { 8. Organizar mi tiempo } \\
\text { para cumplir con todo lo } \\
\text { que los profesores piden }\end{array}$ & ,641 & ,273 & , 142 \\
\hline $\begin{array}{l}\text { 10.Estudiar más horas } \\
\text { cuando tengo pruebas } \\
\text { difíciles }\end{array}$ & ,719 & ,125 & ,080 \\
\hline $\begin{array}{l}\text { 11.Esforzarme mucho } \\
\text { más para resolver tareas } \\
\text { difíciles }\end{array}$ & ,682 & ,305 & , 190 \\
\hline $\begin{array}{l}\text { 12. Repetir una tarea } \\
\text { hasta lograr hacerlo bien }\end{array}$ & ,677 & ,129 & , 159 \\
\hline $\begin{array}{l}\text { 18.Estudiar primero, y } \\
\text { luego hacer otras cosas } \\
\text { (jugar, ver tv) }\end{array}$ & ,692 & ,066 & , 133 \\
\hline $\begin{array}{l}\text { 3. Entender lo que enseña } \\
\text { cualquier profesor }\end{array}$ & , 138 & , 153 & ,725 \\
\hline $\begin{array}{l}\text { 9. Sacarme buenas notas } \\
\text { en las prueba que creo } \\
\text { dificiles }\end{array}$ & ,374 & ,393 & ,450 \\
\hline $\begin{array}{l}\text { 13.Entender bien la idea } \\
\text { central que está } \\
\text { explicando el profesor o } \\
\text { lo que dice un libro }\end{array}$ & ,254 & ,133 & ,641 \\
\hline $\begin{array}{l}17 . \text { Entender lo que } \\
\text { explica un profesor } \\
\text { aunque exista desorden } \\
\text { en la sala. }\end{array}$ & ,050 & ,088 & ,731 \\
\hline
\end{tabular}

\section{Consistencia interna (Alfa de Cronbach)}

Se observa tanto en la tabla $N^{\circ} 5$ y 6 que la escala posee consistencia interna (confiabilidad), lo que explica que los factores están bien relacionados con sus respectivos reactivos. De igual forma, se puede señalar que el instrumento tiene la capacidad para producir resultados congruentes. Cabe destacar que a nivel global la escala presenta un índice de 0,890 siendo adecuada su utilización. 


\begin{tabular}{lcc}
\hline \multicolumn{3}{c}{ Tabla N $^{0}$ 5: Consistencia Interna por factor } \\
\hline Factor & Alfa de Cronbach & N de elementos \\
\hline Confianza en el desempeño de la tarea &, 799 & 8 \\
Esfuerzo en la realización de la tarea &, 800 & 6 \\
Comprensión en la tarea &, 658 & 4 \\
\hline
\end{tabular}

\begin{tabular}{l}
\hline Tabla N $^{\circ}$ 6: Consistencia interna total \\
\hline \multicolumn{3}{|c|}{ Estadísticos de fiabilidad } \\
\begin{tabular}{|r|r|}
\hline $\begin{array}{c}\text { Alfa de } \\
\text { Cronbach }\end{array}$ & $\begin{array}{c}\mathrm{N} \text { de } \\
\text { elementos }\end{array}$ \\
\hline, 890 & 18 \\
\hline
\end{tabular}
\end{tabular}

\section{Correlación Autoeficacia y Rendimiento Académico}

La tabla $\mathrm{N}^{\circ} 7$ muestra la correlación de Sperman entre cada uno de los tres factores y el rendimiento académico de los participantes. En este sentido, se observa a nivel global una correlación significativa y positiva de ,398 entre rendimiento y autoeficacia. Es decir, se aprecia que aquellos estudiantes que muestran un puntaje total más alto en la escala de autoeficacia han obtenido un promedio más alto en rendimiento escolar, durante el período académico 2015. Por su parte, los estudiantes con promedios más bajos tienden hacer una peor evaluación de su autoeficacia escolar. Específicamente, los factores correlacionan significativamente con el rendimiento escolar, siendo de ,365 con confianza en la tarea; un ,332 con esfuerzo en la realización de la tarea y un ,357 con comprensión de la tarea.

\begin{tabular}{|c|c|c|c|c|c|c|c|}
\hline \multicolumn{8}{|c|}{ Tabla N7: Correlaciones Autoeficacia - Rendimiento académico } \\
\hline & & & $\begin{array}{c}\text { Nota } \\
\text { promedio } \\
2015\end{array}$ & $\begin{array}{c}\text { CONFIANZA } \\
\text { EN EL } \\
\text { DESEMPEÑO } \\
\text { DE LA TAREA }\end{array}$ & $\begin{array}{c}\text { ESFUERZO } \\
\text { EN LA } \\
\text { REALIZACIÓN } \\
\text { DE LA TAREA }\end{array}$ & $\begin{array}{l}\text { COMPRE } \\
\text { NSIÓN DE } \\
\text { LA TAREA }\end{array}$ & $\begin{array}{l}\text { PTJE. } \\
\text { TOTAL AE }\end{array}$ \\
\hline \multirow[t]{15}{*}{ Rho de Spearman } & Nota promedio 2015 & $\begin{array}{l}\text { Coeficiente de } \\
\text { correlación }\end{array}$ & 1,000 &, $365^{* *}$ &, $332^{* *}$ &, $357^{* *}$ &, $398^{* *}$ \\
\hline & & Sig. (bilateral) & & ,000 &, 000 & , 000 &, 000 \\
\hline & & $\mathrm{N}$ & 715 & 715 & 715 & 715 & 715 \\
\hline & $\begin{array}{l}\text { CONFIANZA EN EL } \\
\text { DESEMPEÑO DE LA }\end{array}$ & $\begin{array}{l}\text { Coeficiente de } \\
\text { correlación }\end{array}$ &, $365^{\star *}$ & 1,000 &, $664^{* *}$ &, $617^{* *}$ &, $890^{* *}$ \\
\hline & TAREA & Sig. (bilateral) &, 000 & & 000 &, 000 &, 000 \\
\hline & & $\mathrm{N}$ & 715 & 802 & 802 & 802 & 802 \\
\hline & $\begin{array}{l}\text { ESFUERZO EN LA } \\
\text { REALIZACIÓN DE }\end{array}$ & $\begin{array}{l}\text { Coeficiente de } \\
\text { correlación }\end{array}$ &, $332^{* *}$ &, $664^{* *}$ & 1,000 &, $531^{* *}$ &, $874^{\star \star}$ \\
\hline & LA TAREA & Sig. (bilateral) &, 000 & ,000 & &, 000 &, 000 \\
\hline & & $\mathrm{N}$ & 715 & & 802 & & \\
\hline & $\begin{array}{l}\text { COMPRENSIÓN DE } \\
\text { LA TAREA }\end{array}$ & $\begin{array}{l}\text { Coeficiente de } \\
\text { correlación }\end{array}$ &, $357^{* *}$ &, $617^{* *}$ &, $531^{\star *}$ & 1,000 &, $760^{* *}$ \\
\hline & & Sig. (bilateral) & 000 & 000 & 000 & & , 000 \\
\hline & & $\mathrm{N}$ & 715 & 802 & 802 & 802 & 802 \\
\hline & PTJE. TOTAL AE & $\begin{array}{l}\text { Coeficiente de } \\
\text { correlación }\end{array}$ &, $398^{* *}$ &, $890 * *$ &, $874^{* *}$ &, $760^{* *}$ & 1,000 \\
\hline & & Sig. (bilateral) & ,000 & ,000 &, 000 &, 000 & \\
\hline & & $\mathrm{N}$ & 715 & 802 & 802 & 802 & 802 \\
\hline
\end{tabular}




\section{Comparación entre nivel educacional}

La tabla $\mathrm{N}^{\circ} 8$ muestra la estadística descriptiva entre los diferentes ciclos educacionales en relación al nivel de autoeficacia.

\begin{tabular}{|c|c|c|c|c|c|}
\hline \multicolumn{6}{|c|}{ Tabla N8: Estadística descriptiva por ciclo educacional } \\
\hline \multicolumn{6}{|c|}{ Estadísticos de grupo } \\
\hline & Ciclo educacional & $\mathrm{N}$ & Media & $\begin{array}{c}\text { Desviación } \\
\text { típ. }\end{array}$ & $\begin{array}{l}\text { Error típ. de } \\
\text { la media }\end{array}$ \\
\hline \multirow{2}{*}{$\begin{array}{l}\text { 1.Trabajar con cualquier } \\
\text { compañero y lograr } \\
\text { buenas notas }\end{array}$} & Básica & 492 & 3,91 & 879 &, 040 \\
\hline & Media & 309 & 3,93 & ,903 &, 051 \\
\hline \multirow{2}{*}{$\begin{array}{l}\text { 2.Trabajar en cualquier } \\
\text { tarea y lograr buenas } \\
\text { notas }\end{array}$} & Básica & 490 & 3,84 & 900 & ,041 \\
\hline & Media & 309 & & & \\
\hline \multirow{2}{*}{$\begin{array}{l}\text { 4. Realizar bien cualquier } \\
\text { tarea que me den }\end{array}$} & Básica & 483 & 3,89 & 943 & ,043 \\
\hline & Media & 306 & 3,86 & ,854 & ,049 \\
\hline \multirow{2}{*}{$\begin{array}{l}\text { 5.Aportar buenas ideas } \\
\text { para hacer mis tareas en }\end{array}$} & Básica & 480 & 3,62 & 1,121 & ,051 \\
\hline & Media & 307 & 3,63 & 1,082 &, 062 \\
\hline \multirow{2}{*}{$\begin{array}{l}\text { 6. Esforzarme mucho más } \\
\text { que mis compañeros }\end{array}$} & Básica & 487 & 3,71 & 1,088 & ,049 \\
\hline & Media & 309 & 3,57 & 1,060 &, 060 \\
\hline \multirow{2}{*}{$\begin{array}{l}\text { 14.Cooperar muy bien en } \\
\text { los trabajos que realizo }\end{array}$} & Básica & 483 & 4,29 & ,897 & ,041 \\
\hline & Media & 306 & 4,12 & 901 & 052 \\
\hline \multirow{2}{*}{$\begin{array}{l}\text { 15.Estudiar solo/a y rendir } \\
\text { muy bien en cualquier }\end{array}$} & Básica & 486 & 3,77 & 1,106 & ,050 \\
\hline & Media & 309 & 3,86 & 1,000 &, 057 \\
\hline \multirow{2}{*}{$\begin{array}{l}\text { 16.Expresar mi opinión } \\
\text { aunque no esté de }\end{array}$} & Básica & 487 & 3,47 & 1,320 &, 060 \\
\hline & Media & 310 & 3,81 & 1,151 &, 065 \\
\hline \multirow{2}{*}{$\begin{array}{l}\text { CONFIANZA'EN EL"' } \\
\text { DESEMPEÑO DE LA }\end{array}$} & Básica & 492 & 3,8133 & ,66586 &, 03002 \\
\hline & Media & 310 & 3,8356 & 65180 &, 03702 \\
\hline \multirow{2}{*}{$\begin{array}{l}\text { 7.Realizar cualquier tipo } \\
\text { de tarea o trabajo que los }\end{array}$} & Básica & 489 & 3,81 & 1,078 &, 049 \\
\hline & Media & 310 & 3,81 & 1,004 & 057 \\
\hline \multirow{2}{*}{$\begin{array}{l}\text { 8. Organizar mi tiempo } \\
\text { para cumplir con todo lo }\end{array}$} & Básica & 489 & 3,47 & 1,241 &, 056 \\
\hline & Media & 310 & 3,34 & 1,188 & 067 \\
\hline \multirow{2}{*}{$\begin{array}{l}\text { 10.Estudiar más horas } \\
\text { cuando tengo pruebas }\end{array}$} & Básica & 487 & 3,63 & 1,309 &, 059 \\
\hline & Media & 310 & 3,11 & 1,270 & ,072 \\
\hline \multirow{2}{*}{$\begin{array}{l}\text { 11.Esforzarme mucho } \\
\text { más para resolver tareas }\end{array}$} & Básica & 481 & 3,85 & 1,122 & ,051 \\
\hline & Media & 304 & 3,59 & 1,119 & ,064 \\
\hline \multirow{2}{*}{$\begin{array}{l}\text { 12.Repetir una tarea } \\
\text { hasta lograr hacerlo bien }\end{array}$} & Básica & 479 & 3,61 & 1,234 & ,056 \\
\hline & Media & 306 & 3,32 & 1,240 & 071 \\
\hline \multirow{2}{*}{$\begin{array}{l}\text { 18.Estudiar primero, } y \\
\text { luego hacer otras cosas }\end{array}$} & Básica & 490 & 3,47 & 1,407 &, 064 \\
\hline & Media & 308 & 2,97 & 1,289 & ,073 \\
\hline \multirow{2}{*}{$\begin{array}{l}\text { ESFUERZO EN LA } \\
\text { REALIZACIÓN DE LA }\end{array}$} & Básica & 492 & 3,6359 & ,87213 & ,03932 \\
\hline & Media & 310 & 3,3570 & ,83397 & ,04737 \\
\hline \multirow{2}{*}{$\begin{array}{l}\text { 3.Entender lo que enseña } \\
\text { cualquier profesor }\end{array}$} & Básica & 486 & 3,90 &, 945 &, 043 \\
\hline & Media & 308 & 3,81 &, 843 & ,048 \\
\hline \multirow{2}{*}{$\begin{array}{l}\text { 9. Sacarme buenas notas } \\
\text { en las prueba que creo }\end{array}$} & Básica & 489 & 3,52 & 1,103 &, 050 \\
\hline & Media & 307 & 3,36 & 1,020 & ,058 \\
\hline \multirow{2}{*}{$\begin{array}{l}\text { 13.Entender bien la idea } \\
\text { central que está }\end{array}$} & Básica & 489 & 3,92 & 1,024 & ,046 \\
\hline & Media & 308 & 3,79 &, 965 &, 055 \\
\hline \multirow{2}{*}{$\begin{array}{l}\text { 17.Entender lo que } \\
\text { explica un profesor }\end{array}$} & Básica & 486 & 3,27 & 1,370 &, 062 \\
\hline & Media & 309 & 3,34 & 1,195 &, 068 \\
\hline \multirow{2}{*}{$\begin{array}{l}\text { COMPRENSIÓN DE LA } \\
\text { TAREA }\end{array}$} & Básica & 492 & 3,6443 & ,79164 &, 03569 \\
\hline & Media & 310 & 3,5691 &, 71681 & ,04071 \\
\hline PTJE. TOTAL AE & Básica & 492 & 66,1707 & 12,33722 &, 55620 \\
\hline & Media & 310 & 64,7161 & 11,60223 & ,65896 \\
\hline
\end{tabular}

En la tabla $N^{\circ} 9$ se puede apreciar que existen diferencias significativas para 8 de las 18 afirmaciones. El ciclo básico presenta promedios significativamente mayor que el ciclo medio en las siguientes afirmaciones: $N^{0} 14$ "Cooperar muy bien en los trabajos que realizo en grupo"; №16 "Expresar mi opinión aunque no esté de acuerdo con lo que dice el profesor", ambas del factor Confianza en el desempeño de la tarea. También existen diferencias significativas a favor del ciclo básico en las afirmaciones $\mathrm{N}^{\circ} 10$ "Estudiar más horas cuando tengo pruebas difíciles"; N¹1 "Esforzarme mucho más para resolver tareas difíciles"; №12 "Repetir una tarea hasta lograr hacerlo bien" y N018 "Estudiar primero, y luego hacer otras cosas (jugar, ver tv)", todas del factor Esfuerzo en la realización de la tarea, la cual a su conjunto es significativamente 
mayor en el ciclo básico que el ciclo media). Para el factor Comprensión de la tarea, solo la afirmación №9 "Sacarme buenas notas en las pruebas que creo difíciles" el promedio del ciclo básico es significativamente mayor para el ciclo básico. Sólo en la afirmación №2 "Trabajar en cualquier tarea y lograr buenas notas" los estudiantes del ciclo media son mayores que el ciclo básico.

\section{Tabla N09: Significancia estadística}

\begin{tabular}{|c|c|c|c|c|c|c|c|c|c|c|}
\hline & & \multicolumn{2}{|c|}{$\begin{array}{c}\text { Prueba de Levene } \\
\text { para la igualdad de } \\
\text { varianzas }\end{array}$} & \multicolumn{7}{|c|}{ Prueba T para la igualdad de medias } \\
\hline & & \multirow[b]{2}{*}{$\mathrm{F}$} & \multirow[b]{2}{*}{ Sig. } & \multirow[b]{2}{*}{$t$} & \multirow[b]{2}{*}{$\mathrm{gl}$} & \multirow[b]{2}{*}{ Sig. (bilateral) } & \multirow{2}{*}{$\begin{array}{l}\text { Diferencia } \\
\text { de medias }\end{array}$} & \multirow{2}{*}{$\begin{array}{l}\text { Error típ. de } \\
\text { la diferencia }\end{array}$} & \multicolumn{2}{|c|}{$\begin{array}{c}95 \% \text { Intervalo de } \\
\text { confianza para la } \\
\text { diferencia }\end{array}$} \\
\hline 1. Trabajar con cualquier & Se han asumido & & & & & & & & Inferior & Superior \\
\hline $\begin{array}{l}\text { compañero y lograr } \\
\text { buenas notas }\end{array}$ & $\begin{array}{l}\text { varianzas iguales } \\
\text { No se han asumido } \\
\text { varianzas iguales }\end{array}$ & 233 & 629 & $\begin{array}{l}-, 201 \\
-, 200\end{array}$ & $\begin{array}{r}799 \\
640,996\end{array}$ & $\begin{array}{l}, 841 \\
, 842\end{array}$ & $\begin{array}{l}-, 013 \\
-, 013\end{array}$ & $\begin{array}{l}, 064 \\
, 065\end{array}$ & $\begin{array}{l}-, 140 \\
-, 140\end{array}$ & $\begin{array}{l}, 114 \\
, 114\end{array}$ \\
\hline $\begin{array}{l}\text { 2. Trabajar en cualquier } \\
\text { tarea y lograr buenas } \\
\text { notas }\end{array}$ & $\begin{array}{l}\text { Se han asumido } \\
\text { varianzas iguales } \\
\text { No se han asumido } \\
\text { varianzas iguales }\end{array}$ & 4,305 & 038 & $\begin{array}{l}-1,153 \\
-1,165\end{array}$ & $\begin{array}{r}797 \\
675,795\end{array}$ & $\begin{array}{l}, 249 \\
, 245\end{array}$ & $\begin{array}{l}-, 074 \\
-, 074\end{array}$ & $\begin{array}{l}, 064 \\
, 064\end{array}$ & $\begin{array}{l}-, 200 \\
-, 199\end{array}$ &, 051 \\
\hline $\begin{array}{l}\text { 4. Realizar bien cualquier } \\
\text { tarea que me den }\end{array}$ & $\begin{array}{l}\text { Se han asumido } \\
\text { varianzas iguales } \\
\text { No se han asumido } \\
\text { varianzas iguales }\end{array}$ & 3,481 &, 062 & $\begin{array}{l}, 445 \\
, 455\end{array}$ & $\begin{array}{r}787 \\
695,662\end{array}$ & $\begin{array}{l}, 656 \\
, 649\end{array}$ & $\begin{array}{l}, 030 \\
, 030\end{array}$ & $\begin{array}{l}, 066 \\
, 065\end{array}$ & $\begin{array}{l}-, 101 \\
-, 098\end{array}$ & $\begin{array}{l}, 160 \\
, 157\end{array}$ \\
\hline $\begin{array}{l}\text { 5.Aportar buenas ideas } \\
\text { para hacer mis tareas en } \\
\text { todos los ramos }\end{array}$ & $\begin{array}{l}\text { Se han asumido } \\
\text { varianzas iguales } \\
\text { No se han asumido } \\
\text { varianzas iguales }\end{array}$ & 804 & 370 & $\begin{array}{l}-, 108 \\
-, 109\end{array}$ & $\begin{array}{r}785 \\
668,986\end{array}$ & $\begin{array}{l}, 914 \\
, 913\end{array}$ & $\begin{array}{r}-, 009 \\
-, 009\end{array}$ & $\begin{array}{l}, 081 \\
, 080\end{array}$ & $\begin{array}{l}-, 167 \\
-, 166\end{array}$ & , 149 \\
\hline $\begin{array}{l}\text { 6. Esforzarme mucho más } \\
\text { que mis compañeros } \\
\text { para que me vaya bien } \\
\text { en todos los ramos }\end{array}$ & $\begin{array}{l}\text { Se han asumido } \\
\text { varianzas iguales } \\
\text { No se han asumido } \\
\text { varianzas iguales }\end{array}$ &, 040 & 842 & $\begin{array}{l}1,824 \\
1,835\end{array}$ & $\begin{array}{r}794 \\
668,590\end{array}$ & $\begin{array}{l}, 068 \\
, 067\end{array}$ & $\begin{array}{l}, 143 \\
, 143\end{array}$ & $\begin{array}{l}, 078 \\
, 078\end{array}$ & $\begin{array}{r}-, 011 \\
-, 010\end{array}$ & $\begin{array}{l}, 297 \\
, 296\end{array}$ \\
\hline $\begin{array}{l}\text { 14. Cooperar muy bien en } \\
\text { los trabajos que realizo } \\
\text { en grupo. }\end{array}$ & $\begin{array}{l}\text { Se han asumido } \\
\text { varianzas iguales } \\
\text { No se han asumido } \\
\text { varianzas iguales }\end{array}$ & 1,264 & 261 & $\begin{array}{l}2,654 \\
2,651\end{array}$ & $\begin{array}{r}787 \\
646,744\end{array}$ & $\begin{array}{l}, 008 \\
, 008\end{array}$ & $\begin{array}{l}, 174 \\
, 174\end{array}$ & $\begin{array}{l}.066 \\
, 066\end{array}$ & $\begin{array}{l}, 045 \\
, 045\end{array}$ & $\begin{array}{l}, 303 \\
, 303\end{array}$ \\
\hline $\begin{array}{l}\text { 15.Estudiar solo/a y rendir } \\
\text { muy bien en cualquier } \\
\text { actividad académica }\end{array}$ & $\begin{array}{l}\text { Se han asumido } \\
\text { varianzas iguales } \\
\text { No se han asumido } \\
\text { varianzas iguales }\end{array}$ & 6,574 & 011 & $\begin{array}{l}-1,188 \\
-1,216\end{array}$ & $\begin{array}{r}793 \\
703,440\end{array}$ & $\begin{array}{l}, 235 \\
, 225\end{array}$ & $\begin{array}{r}-, 092 \\
-, 092\end{array}$ & $\begin{array}{l}.078 \\
.076\end{array}$ & $\begin{array}{r}-, 244 \\
-, 241\end{array}$ & ,057 \\
\hline $\begin{array}{l}\text { 16. Expresar mi opinión } \\
\text { aunque no esté de } \\
\text { acuerdo con lo que dice } \\
\text { el profesor }\end{array}$ & $\begin{array}{l}\text { Se han asumido } \\
\text { varianzas iguales } \\
\text { No se han asumido } \\
\text { varianzas iguales }\end{array}$ & 19,176 & 000 & $\begin{array}{l}-3,752 \\
-3,867\end{array}$ & $\begin{array}{r}795 \\
721,701\end{array}$ & $\begin{array}{l}, 000 \\
, 000\end{array}$ & $\begin{array}{l}-, 343 \\
-, 343\end{array}$ & $\begin{array}{l}, 091 \\
, 089\end{array}$ & $\begin{array}{l}-, 522 \\
-, 517\end{array}$ & $\begin{array}{l}-, 163 \\
-, 169\end{array}$ \\
\hline $\begin{array}{l}\text { CONFIANZA EN EL } \\
\text { DESEMPENOO DE LA } \\
\text { TAREA }\end{array}$ & $\begin{array}{l}\text { Se han asumido } \\
\text { varianzas iguales } \\
\text { No se han asumido } \\
\text { varianzas iguales }\end{array}$ & 1,024 &, 312 & $\begin{array}{r}-, 466 \\
-, 468\end{array}$ & $\begin{array}{r}800 \\
667,382\end{array}$ & $\begin{array}{l}, 641 \\
, 640\end{array}$ & $\begin{array}{r}-, 02233 \\
-, 02233\end{array}$ & $\begin{array}{l}, 04789 \\
, 04766\end{array}$ & $\begin{array}{l}-, 11634 \\
-, 11591\end{array}$ & $\begin{array}{l}.07168 \\
.07126\end{array}$ \\
\hline $\begin{array}{l}\text { 7. Realizar cualquier tipo } \\
\text { de tarea o trabajo que los } \\
\text { profesores den, aunque } \\
\text { sean difíciles }\end{array}$ & $\begin{array}{l}\text { Se han asumido } \\
\text { varianzas iguales } \\
\text { No se han asumido } \\
\text { varianzas iguales }\end{array}$ & 1,453 & 228 & $\begin{array}{l}, 002 \\
, 002\end{array}$ & $\begin{array}{r}797 \\
691,618\end{array}$ & $\begin{array}{l}, 999 \\
, 999\end{array}$ & $\begin{array}{l}, 000 \\
, 000\end{array}$ & $\begin{array}{l}, 076 \\
, 075\end{array}$ & $\begin{array}{r}-, 150 \\
-, 147\end{array}$ & $\begin{array}{l}, 150 \\
, 147\end{array}$ \\
\hline $\begin{array}{l}\text { 8. Organizar mi tiempo } \\
\text { para cumplir con todo lo } \\
\text { que los profesores piden }\end{array}$ & $\begin{array}{l}\text { Se han asumido } \\
\text { varianzas iguales } \\
\text { No se han asumido } \\
\text { varianzas iguales }\end{array}$ &, 511 &, 475 & $\begin{array}{l}1,472 \\
1,487\end{array}$ & $\begin{array}{r}797 \\
679,017\end{array}$ & $\begin{array}{l}, 141 \\
, 138\end{array}$ & $\begin{array}{l}, 130 \\
, 130\end{array}$ & $\begin{array}{l}.089 \\
.088\end{array}$ & $\begin{array}{r}-, 044 \\
-, 042\end{array}$ & $\begin{array}{l}, 304 \\
, 303\end{array}$ \\
\hline $\begin{array}{l}\text { 10. Estudiar más horas } \\
\text { cuando tengo pruebas } \\
\text { dificiles }\end{array}$ & $\begin{array}{l}\text { Se han asumido } \\
\text { varianzas iguales } \\
\text { No se han asumido } \\
\text { varianzas iguales }\end{array}$ & ,115 &, 734 & $\begin{array}{l}5,560 \\
5,598\end{array}$ & $\begin{array}{r}795 \\
672,836\end{array}$ & $\begin{array}{l}, 000 \\
, 000\end{array}$ & $\begin{array}{l}, 523 \\
, 523\end{array}$ & $\begin{array}{l}, 094 \\
, 093\end{array}$ & $\begin{array}{l}, 338 \\
, 339\end{array}$ & $\begin{array}{l}, 707 \\
, 706\end{array}$ \\
\hline $\begin{array}{l}\text { 11.Esforzarme mucho } \\
\text { más para resolver tareas } \\
\text { dificiles }\end{array}$ & $\begin{array}{l}\text { Se han asumido } \\
\text { varianzas iguales } \\
\text { No se han asumido } \\
\text { varianzas iguales }\end{array}$ & 854 & 356 & $\begin{array}{l}3,195 \\
3,197\end{array}$ & $\begin{array}{r}783 \\
645,612\end{array}$ & $\begin{array}{l}, 001 \\
, 001\end{array}$ & $\begin{array}{l}, 262 \\
, 262\end{array}$ & $\begin{array}{l}, 082 \\
, 082\end{array}$ & $\begin{array}{l}, 101 \\
, 101\end{array}$ &, 424 \\
\hline $\begin{array}{l}\text { 12. Repetir una tarea } \\
\text { hasta lograr hacerlo bien }\end{array}$ & $\begin{array}{l}\text { Se han asumido } \\
\text { varianzas iguales } \\
\text { No se han asumido } \\
\text { varianzas iguales }\end{array}$ & 688 & 407 & $\begin{array}{l}3,175 \\
3,172\end{array}$ & $\begin{array}{r}783 \\
647,897\end{array}$ & $\begin{array}{l}, 002 \\
, 002\end{array}$ & $\begin{array}{l}, 287 \\
, 287\end{array}$ & $\begin{array}{l}, 090 \\
, 091\end{array}$ & $\begin{array}{l}, 110 \\
, 109\end{array}$ &, 465 \\
\hline $\begin{array}{l}\text { 18. Estudiar primero, y } \\
\text { luego hacer otras cosas } \\
\text { (jugar, ver tv) }\end{array}$ & $\begin{array}{l}\text { Se han asumido } \\
\text { varianzas iguales } \\
\text { No se han asumido } \\
\text { varianzas iguales }\end{array}$ & 5,544 & 019 & $\begin{array}{l}5,041 \\
5,143\end{array}$ & $\begin{array}{r}796 \\
694,642\end{array}$ & $\begin{array}{l}, 000 \\
, 000\end{array}$ & $\begin{array}{l}.499 \\
, 499\end{array}$ & $\begin{array}{l}.099 \\
.097\end{array}$ & $\begin{array}{l}, 305 \\
, 309\end{array}$ & 694 \\
\hline $\begin{array}{l}\text { ESFUERZO EN LA } \\
\text { REALIZACIÓN DE LA } \\
\text { TAREA }\end{array}$ & $\begin{array}{l}\text { Se han asumido } \\
\text { varianzas iguales } \\
\text { No se han asumido } \\
\text { varianzas iguales }\end{array}$ &, 727 & ,394 & 4,531 & $\begin{array}{r}800 \\
678,747\end{array}$ &, 000 & $\begin{array}{l}, 27891 \\
, 27891\end{array}$ & $\begin{array}{l}, 06219 \\
, 06156\end{array}$ & $\begin{array}{l}, 15684 \\
, 15804\end{array}$ & $\begin{array}{l}, 40098 \\
, 39978\end{array}$ \\
\hline $\begin{array}{l}\text { 3. Entender lo que enseña } \\
\text { cualquier profesor }\end{array}$ & $\begin{array}{l}\text { Se han asumido } \\
\text { varianzas iguales } \\
\text { No se han asumido } \\
\text { varianzas iguales }\end{array}$ & ,988 &, 320 & 1,392 & $\begin{array}{r}792 \\
706,619\end{array}$ & $\begin{array}{l}, 164 \\
, 154\end{array}$ & $\begin{array}{l}, 092 \\
, 092\end{array}$ & $\begin{array}{l}, 066 \\
, 064\end{array}$ & $\begin{array}{r}-, 038 \\
-, 034\end{array}$ & $\begin{array}{l}, 222 \\
, 218\end{array}$ \\
\hline $\begin{array}{l}\text { 9. Sacarme buenas notas } \\
\text { en las prueba que creo } \\
\text { dificiles }\end{array}$ & $\begin{array}{l}\text { Se han asumido } \\
\text { varianzas iguales } \\
\text { No se han asumido } \\
\text { varianzas iguales }\end{array}$ & 1,959 & 162 & $\begin{array}{l}2,080 \\
2,117\end{array}$ & $\begin{array}{r}794 \\
687,982\end{array}$ & $\begin{array}{l}, 038 \\
, 035\end{array}$ & $\begin{array}{l}, 162 \\
, 162\end{array}$ & $\begin{array}{l}, 078 \\
, 077\end{array}$ & $\begin{array}{l}, 009 \\
, 012\end{array}$ & $\begin{array}{l}, 316 \\
, 313\end{array}$ \\
\hline $\begin{array}{l}\text { 13.Entender bien la idea } \\
\text { central que está } \\
\text { explicando el profesor o } \\
\text { lo que dice un libro }\end{array}$ & $\begin{array}{l}\text { Se han asumido } \\
\text { varianzas iguales } \\
\text { No se han asumido } \\
\text { varianzas iguales }\end{array}$ & 380 &, 538 & 1,815 & $\begin{array}{r}795 \\
681,257\end{array}$ & ,070 & , 130 & , 072 & $\begin{array}{r}-, 013 \\
-, 011\end{array}$ & ,272 \\
\hline $\begin{array}{l}\text { 17.Entender lo que } \\
\text { explica un profesor } \\
\text { aunque exista desorden } \\
\text { en la sala. }\end{array}$ & $\begin{array}{l}\text { Se han asumido } \\
\text { varianzas iguales } \\
\text { No se han asumido } \\
\text { varianzas iguales }\end{array}$ & 16,497 &, 000 & $\begin{array}{r}-, 817 \\
-, 843\end{array}$ & $\begin{array}{r}793 \\
719,197\end{array}$ &, 414 & $\begin{array}{r}-, 078 \\
-, 078\end{array}$ & ,095 &,- 264 & , 109 \\
\hline $\begin{array}{l}\text { COMPRENSIÓN DE LA } \\
\text { TAREA }\end{array}$ & $\begin{array}{l}\text { Se han asumido } \\
\text { varianzas iguales } \\
\text { No se han asumido } \\
\text { varianzas iguales }\end{array}$ & 3,072 & 080 & $\begin{array}{l}1,357 \\
1,388\end{array}$ & $\begin{array}{r}800 \\
704,560\end{array}$ & , 166 & $\begin{array}{l}, 07516 \\
, 07516\end{array}$ & $\begin{array}{l}, 05537 \\
, 05414\end{array}$ & $\begin{array}{l}-, 03353 \\
-, 03114\end{array}$ & , 18146 \\
\hline PTJE. TOTAL AE & $\begin{array}{l}\text { Se han asumido } \\
\text { varianzas iguales } \\
\text { No se han asumido } \\
\text { varianzas iguales }\end{array}$ & 1,861 & 173 & $\begin{array}{l}1,663 \\
1,687\end{array}$ & $\begin{array}{r}800 \\
686,758\end{array}$ & 097 & 1,45460 & ,87442 & $\begin{array}{l}-, 26183 \\
-, 23850\end{array}$ & 3,17104 \\
\hline
\end{tabular}




\section{Conclusiones}

El análisis factorial realizado a la escala de autoeficacia escolar (ACAES) fundamenta la idea de un constructo de 3 factores que en conjunto explican cerca del $50 \%$ de la varianza. A nivel de confiabilidad se obtuvo un índice $(, 89)$ el cual es considerado adecuado en la teoría psicométrica. Es decir, los ítemes de la escala pueden ser considerados homogéneos, en el sentido que miden lo mismo o representan un mismo dominio empírico, a saber, autoeficacia escolar.

En este sentido, el comportamiento estadístico de la escala en la muestra de estudiantes de este estudio, ratifica lo planteado por Bandura (1997) en relación con la medida de la autoeficacia. Este autor sugiere a los investigadores que "dichas creencias debieran ser medidas en términos de juicios específicos de capacidad, sometidos a variaciones en función de la actividad, las exigencias de la tarea y otras múltiples circunstancias situacionales". Por tanto, el instrumento utilizado en el presente estudio valida los datos obtenidos.

A nivel del análisis correlacional, los hallazgos del estudio se mantienen en la línea de los estudios de Bandura (1995) toda vez que plantea que las creencias de autoeficacia, entre ellas las creencias de los estudiantes respecto a su eficacia para regular su propio aprendizaje, son un mediador cognitivo en el logro de aprendizajes. En este sentido, se torna fundamental la idea de promover prácticas de autoregulación que permitan a los estudiantes tomar conciencia de sus procesos de pensamiento y la forma de apoyar el desarrollo de la autoeficacia.

De igual forma, las relaciones encontradas entre autoeficacia y rendimiento escolar vienen a corroborar los hallazgos realizados por Contreras et al. (2005) quienes demostraron que la autoeficacia resulta predictiva del logro académico; afectando la conducta del individuo en las elecciones que realiza, el esfuerzo que aplica, la perseverancia ante los obstáculos con que se enfrenta, los patrones de pensamiento y las reacciones emocionales que experimenta.

Al comparar la autoeficacia por ciclo educacional (enseñanza básica y media), no se observan diferencias tanto a nivel de la escala total como dos de sus factores. No obstante, la diferencias significativas encontradas, principalmente a nivel del factor "Esfuerzo en la realización de la tarea", se puede hipotetizar respecto a que los estudiantes de la muestra cursan entre el $5^{\circ}$ y $8^{\circ}$ de enseñanza básica y cuyas edades pueden fluctuar entre los 10 y 13, etapa donde el nivel de autoeficacia es relativamente normal y no presenta disminuciones significativas como en la etapa de la adolescencia, etapa del desarrollo que se encuentran los alumnos que cursan la enseñanza media. En este sentido, Bandura (1997) plantea que la adolescencia es una etapa de transición y turbulencias en la que cabe esperar un descenso de la autoeficacia. Por su parte, Carrasco y Del Barrio (2002) señalan que los cambios en el dominio académico pueden guardar relevancia con los desafíos a los que se enfrentan los jóvenes, lo que haría comprensible que haya un descenso y modificación de la autoeficacia a medida que se ingresa a esta etapa de desarrollo.

Referencias

Bandura, A. (1977). Self-efficacy: Toward a unifying theory of behavioral change. Psychological Review, 84(2), 191-215.

Bandura, A. (1982). Teoría del Aprendizaje Social. Madrid: Espasa Calpe.
Bandura, A. (1986). Social foundations of thought and action: A social cognitive theory. Englewood Cliffs, NJ: Prentice-Hall.

Bandura, A. (1987). Pensamiento y acción. Fundamentos sociales. Barcelona: Martínez Roca. 
Bandura, A. (1993). Perceived self-efficacy in cognitive development and functioning . Educational Psychologist, 28, 117-148.

Bandura, A. (1995). Self-Efficacy in changing societies. Cambridge: University Press.

Bandura, A. (1997). Self-efficacy: The exercise of control. New York: Freeman.

Bandura, A. (1999). Auto-eficacia: Cómo afrontamos los cambios de la sociedad actual. Biblioteca de psicología Bilbao, España: Desclée De Bower.

Bandura, A. (2001). Guía para la construcción de Escalas de Autoeficacia.(versión revisada). EEUU: Universidad The Stanford.

Bardales, K., Díaz, P., Terrenos, M., \& Valencia, M. (2006). Psicología social : pasadp, presente y futuro. Universidad del Valle: Instituto de Psicología.

Camposeco, F. d. (2012). La autoeficaciacomo como variable en la motivación intrínseca y extrínsecaen matemáticas a través de un criterio étnico. Madrid: Memoria para optar al grado de doctor por la Universidad Complutense de Madrid.

Carrasco, M., \& Del Barrio, M. (2002). Evaluación de la autoeficacia en niños y adolescentes. Psicothema, Vol. 14(2), 323-332.

Cartagena, M. (2008). Relación entre autoeficacia y rendimiento académico y los hábitos de estudios en alumnos secundaria.
Revista Iberoamericana sobre Calidad, Eficacia y Cambio en Educación, Volumen 6, Número 3.

Contreras, F., Espinosa, J., Esguerra, G., Haikal, A., Polanía, A., \& Rodríguez, A. (2005). Autoeficacia, ansiedad y rendimiento académico en adolescentes. Perspectivas en Psicología, 183194.

González Fernández, A. (2005). Motivación académica. Teoría, aplicación y evaluación. Madrid: Pirámide.

Pajares, F., \& Schunk, D. (2001). Self-Beliefs and School Success: Self-Efficacy, Self-Concept, and School Achievement. In R. Riding y S. Rayner (Eds.), Perception, pp. 239-266 Ablex Publishing, London.

Paoloni, P., \& Bonetto, V. (2013). Creencias de autoeficacia y rendimiento académico en estudiantes universitarios. Recuperado el 15 de Mayo de 2016, de http://www.psicologiacientifica.com/creencias-de-autoeficaciay-rendimiento-academico

Serra, J. (2010). Autoeficacia y Rendimiento Académico en Estudiantes Universitarios. Revista Griot, Volumen 3, Número. 2.

Velásquez, A. (2012). Revisión histórico conceptual del concepto de autoeficacia. Revista Pequén, 148-160.

Zimmerman, B. (1995). Self-efficacy and educational development. In A. Bandura (Ed.), Self-efficacy in changing societies (pp. 202-231). New York: Cambridge Univ. Press.

\section{Revisión por pares abierta | Open Peer Review}

Dra. Karen McMullin. Trent University (Canada)

0000-0002-1449-3550

Dra. Marzia Fiorini. Università degli Studi di Cassino e del Lazio Meridionale (Italia)

- 778877 


\section{APÉNDICE}

\section{ESCALA DE AUTOEFICACIA ACADÉMICA DE LOS ESCOLARES (ACAES)}

El presente instrumento tiene por finalidad conocer la percepción que tienes como estudiante respecto a tus capacidades para realizar con éxito las actividades académicas, como por ejemplo: Pruebas, Tareas, Ejercicios, Proyectos, Presentaciones o cualquier otra actividad que establezca el colegio.

No hay preguntas buenas o malas. Por lo tanto, te pedimos responderlas todas.

Tus respuestas serán analizadas en forma confidencial y por eso no te pedimos tu nombre.

Para responder este instrumento, deberás leer cada una de las afirmaciones anteponiendo un "YO PUEDO". Por ejemplo, si la afirmación dice: "Trabajar con cualquier compañero y lograr buenas notas", deberá leerse "YO PUEDO trabajar con cualquier compañero y lograr buenas notas".

Posteriormente, deberás marcar con una " $\mathrm{X}$ " (encima del número) solo en aquel valor que más se acerca a tu realidad, siguiendo esta escala:

\begin{tabular}{|l|l|l|l|l|}
\hline Nunca puedo $=1$ & Casi nunca puedo $=2$ & No sé qué responder $=3$ & Casiempre puedo $=4$ \\
\hline
\end{tabular}

Te solicitamos indicarnos el promedio final de notas que obtuviste en el año 2015 y el curso actual en el año 2016.

\begin{tabular}{|c|c|c|}
\hline PROMEDIO DE NOTAS & CURSO ACTUAL \\
2015 & 2016 \\
\hline
\end{tabular}

\begin{tabular}{|c|c|c|c|c|c|}
\hline $\begin{array}{c}\text { RECUERDA: Cuando leas cada afirmaciones deberás anteponer } \\
\text { lo siguiente: YO PUEDO }\end{array}$ & $\begin{array}{l}\text { Nunca } \\
\text { puedo }\end{array}$ & $\begin{array}{c}\text { Casi } \\
\text { nunca } \\
\text { puedo }\end{array}$ & $\begin{array}{l}\text { No sé } \\
\text { qué } \\
\text { responder }\end{array}$ & $\begin{array}{l}\text { Casi } \\
\text { siempre } \\
\text { puedo }\end{array}$ & $\begin{array}{l}\text { Siempre } \\
\text { puedo }\end{array}$ \\
\hline Trabajar con cualquier compañero y lograr buenas notas & 1 & 2 & 3 & 4 & 5 \\
\hline Trabajar en cualquier tarea y lograr buenas notas & 1 & 2 & 3 & 4 & 5 \\
\hline Entender lo que enseña cualquier profesor & 1 & 2 & 3 & 4 & 5 \\
\hline 4. Realizar bien cualquier tarea que me den & 1 & 2 & 3 & 4 & 5 \\
\hline $\begin{array}{l}\text { 5. Aportar buenas ideas para hacer mis tareas en todos los } \\
\text { ramos }\end{array}$ & 1 & 2 & 3 & 4 & 5 \\
\hline $\begin{array}{l}\text { 6. Esforzarme mucho más que mis compañeros para que me } \\
\text { vaya bien en todos los ramos }\end{array}$ & 1 & 2 & 3 & 4 & 5 \\
\hline $\begin{array}{l}\text { 7. Realizar cualquier tipo de tarea o trabajo que los profesores } \\
\text { den, aunque sean difíciles }\end{array}$ & 1 & 2 & 3 & 4 & 5 \\
\hline $\begin{array}{l}\text { 8. Organizar mi tiempo para cumplir con todo lo que los } \\
\text { profesores piden }\end{array}$ & 1 & 2 & 3 & 4 & 5 \\
\hline 9. Sacarme buenas notas en las prueba que creo difíciles & 1 & 2 & 3 & 4 & 5 \\
\hline 10. Estudiar más horas cuando tengo pruebas difíciles & 1 & 2 & 3 & 4 & 5 \\
\hline 11. Esforzarme mucho más para resolver tareas difíciles & 1 & 2 & 3 & 4 & 5 \\
\hline 12. Repetir una tarea hasta lograr hacerlo bien & 1 & 2 & 3 & 4 & 5 \\
\hline $\begin{array}{l}\text { 13. Entender bien la idea central que está explicando el profesor } \\
\text { o lo que dice un libro }\end{array}$ & 1 & 2 & 3 & 4 & 5 \\
\hline 14. Cooperar muy bien en los trabajos que realizo en grupo. & 1 & 2 & 3 & 4 & 5 \\
\hline $\begin{array}{l}\text { 15. Estudiar solo/a y rendir muy bien en cualquier actividad } \\
\text { académica }\end{array}$ & 1 & 2 & 3 & 4 & 5 \\
\hline $\begin{array}{l}\text { 16. Expresar mi opinión aunque no esté de acuerdo con lo que } \\
\text { dice el profesor }\end{array}$ & 1 & 2 & 3 & 4 & 5 \\
\hline $\begin{array}{l}\text { 17. Entender lo que explica un profesor aunque exista desorden } \\
\text { en la sala. }\end{array}$ & 1 & 2 & 3 & 4 & 5 \\
\hline 18. Estudiar primero, y luego hacer otras cosas (jugar, ver tv) & 1 & 2 & 3 & 4 & 5 \\
\hline
\end{tabular}

